\title{
Cluster analysis of immunohistochemical profiles in synovial sarcoma, malignant peripheral nerve sheath tumor, and Ewing sarcoma
}

\author{
Stephen H Olsen, Dafydd G Thomas and David R Lucas \\ Department of Pathology, The University of Michigan Medical School, Ann Arbor, MI, USA
}

\begin{abstract}
As a result of overlapping morphologic and immunohistochemical features, it can be difficult to distinguish synovial sarcoma, malignant peripheral nerve sheath tumor, and Ewing sarcoma/primitive neuroectodermal tumor in core biopsies. To analyze and compare immunohistochemical profiles, we stained tissue microarrays of 23 synovial sarcomas, 23 malignant peripheral nerve sheath tumors, and 27 Ewing sarcomas with 22 antibodies potentially useful in the differential diagnosis, and analyzed the data with cluster analysis. Stain intensity was scored as none, weak, or strong. For CD99, tumors with membranous accentuation were independently categorized. Cluster analysis sorted five groups, with like tumors clustering together. Synovial sarcoma clustered into two groups: one cytokeratin and EMA positive $(n=11)$, the other mostly cytokeratin negative, EMA positive, bcl-2 positive and mostly CD56 positive $(n=9)$. Malignant peripheral nerve sheath tumor clustered into two groups: one S100 positive, with nestin and NGFR positivity in most $(n=10)$, the other mostly S100 negative, and variably but mostly weakly positive for nestin and NGFR $(n=11)$. Ewing sarcomas clustered into a single group driven by membranous CD99 staining. Thirteen cases failed to cluster (outliers), while three Ewing sarcomas clustered into groups of other tumor types. Paired antibodies for each tumor type determined by visual assessment of cluster analysis data and statistical calculations of specificity, sensitivity, and predictive values showed that EMA/CK7 for synovial sarcoma, nestin/S100 for malignant peripheral nerve sheath tumor, and membranous CD99/Fli-1 for Ewing sarcoma yielded high specificity and positive predictive values. Cluster analysis also highlighted aberrant staining reactions and diagnostic pitfalls in these tumors. Hierarchical cluster analysis is an effective method for analyzing high-volume immunohistochemical data. Modern Pathology (2006) 19, 659-668. doi:10.1038/modpathol.3800569; published online 10 March 2006
\end{abstract}

Keywords: immunohistochemistry; cluster analysis; tissue microarray; sarcoma

Advancements in image-guided needle core biopsy technique have resulted in less invasive procedures compared to traditional open biopsy. CT or ultrasound-guided core biopsy is now the standard diagnostic test for soft tissue tumors in many cancer centers, including the University of Michigan. Although core biopsy is associated with reduced morbidity and greater convenience, the small sample size has made histological diagnosis more challenging. This is especially true for tumors with

Correspondence: Dr DR Lucas, MD, Department of Pathology, The University of Michigan Medical School, 1500 E. Medical Center Drive, Room 2G332 UH, Ann Arbor, MI 48109-0054, USA.

E-mail: drlucas@umich.edu

The results of this study were presented in part at the 94th Annual Meeting of the United States and Canadian Academy of Pathologists in San Antonio, TX, February 2005.

Received 27 September 2005; revised and accepted 17 January 2006; published online 10 March 2006 overlapping morphologic features, such as synovial sarcoma, malignant peripheral nerve sheath tumor, and Ewing sarcoma/primitive neuroectodermal tumor; specifically, closely spaced, hyperchromatic cells with frequent ovoid to round nuclear contours. Advancements in immunohistochemistry in soft tissue sarcomas have ameliorated this problem to some extent. However, limitations in sensitivity and specificity for many of the common markers continue to be problematic.

Cytokeratin, S100, and CD99 are typically regarded as the most useful single markers for synovial sarcoma, malignant peripheral nerve sheath tumor, and Ewing sarcoma, respectively. However, they are not always reliable. For example, cytokeratin, although positive in most biphasic synovial sarcomas, is less likely to stain monophasic and poorly differentiated synovial sarcomas in small samples. ${ }^{1-3}$ In addition, cytokeratin can be found in 
a minority of malignant peripheral nerve sheath tumors $^{4}$ and Ewing sarcomas., S100 protein is positive in only around half of malignant peripheral nerve sheath tumors, ${ }^{7}$ however, can also stain synovial sarcoma ${ }^{4,8}$ and Ewing sarcoma, ${ }^{9}$ and thus lacks sufficient sensitivity and specificity to be a very reliable marker. Finally, CD99, although positive in $>90 \%{ }^{10-12}$ of Ewing sarcomas, lacks specificity since it stains other mesenchymal ${ }^{13}$ and lymphoid neoplasms, ${ }^{11}$ including frequent expression in synovial sarcoma. ${ }^{1,9}$ As a result of the broad spectrum of CD99-positive tumors, it has been proposed that membranous accentuation be regarded a more specific staining reaction for Ewing sarcoma. ${ }^{10}$

A number of other antibodies have been touted as useful for these tumor types. For example, cytokeratin subsets, especially CK7 and $19,,^{3,9,14}$ bcl- $2,^{15}$ CD56, ${ }^{9}$ calponin, ${ }^{16}$ beta-catenin, ${ }^{17}$ and matrix metalloproteinase-2 (MMP-2) ${ }^{18}$ have been reported as potentially useful in synovial sarcoma; p75 nerve growth factor receptor (NGFR), ${ }^{19}$ nestin, ${ }^{20}$ CD57, ${ }^{7,21,22}$ CD34, ${ }^{22}$ PGP9.5, ${ }^{23}$ and GFAP ${ }^{21}$ in malignant peripheral nerve sheath tumor; Fli-1 in Ewing sarcoma $;{ }^{6}$ and neuroectodermal markers such as chromoganin, synaptophysin, and PGP9.5 in primitive neuroectodermal tumor. ${ }^{24-27}$

In order to further characterize the immunohistochemical profiles of synovial sarcoma, malignant peripheral nerve sheath tumor, and Ewing sarcoma in a model that mimics small biopsy sampling, we evaluated a series of 73 tumors in tissue microarrays with a panel of 22 antibodies potentially useful in the differential diagnosis. We then analyzed the data with hierarchical cluster analysis, a mathematical technique employed for analyzing large data sets, most often used for gene arrays.

\section{Materials and methods}

Tumors were identified via SNOMED search of the pathology database, and 23 synovial sarcomas, 23 malignant peripheral nerve sheath tumors, and 27 Ewing sarcomas were selected, otherwise randomly, based on available tumor volume. All tumors were diagnosed by conventional methodology. Synovial sarcoma diagnoses were confirmed by RT-PCR for $S Y T / S S X$ in every case, ${ }^{28}$ and the tumors were subcategorized as biphasic or monophasic based upon review of the original sections. Malignant peripheral nerve sheath tumors were subclassified as spindle cell, epithelioid, pleomorphic or small cell types based upon predominant histologic element. From the clinical record, each malignant peripheral nerve sheath tumor was categorized as either sporadic or neurofibromatosis associated. Tissue microarray slides from the Ewing sarcoma cases were analyzed by fluorescence in-situ hybridization (FISH) using a dual-color break-apart probe cocktail flanking the EWS-R1 breakpoint region (22q12) (Vysis, Downers Grove, IL, USA). ${ }^{29}$ Tissue microarray blocks were constructed from $1 \mathrm{~mm}$ cores of formalin-fixed, paraffin-embedded tissue in triplicate. One tissue microarray block was produced for each tumor type, with each block containing a variety of normal and neoplastic tissue samples as internal controls.

The slides were stained with a panel of 22 antibodies by standard immunohistochemical techniques. Antibody information with pretreatments and incubation times are listed in Table 1. Intensity of staining was scored as negative, weak, or strong with attention to distribution and cellular localization of staining. For CD99, the staining reaction was categorized as generalized for any cytosolic or

Table 1 Antibody information

\begin{tabular}{|c|c|c|c|c|c|}
\hline Antibody & Source & City, State & Pretreatment & Dilution & Incubation (min) \\
\hline PGP9.5 & DAKO & Carpinteria, CA & HIER & $1: 200$ & 30 \\
\hline Fli-1 & Neomarkers & Fremont, CA & HIER & $1: 500$ & 60 \\
\hline Nestin & SCBT & Santa Cruz, CA & HIER & $1: 500$ & 60 \\
\hline GFAP & Novocastra & Newcastle, UK & HIER & $1: 100$ & 60 \\
\hline S100 & DAKO & Carpinteria, CA & None & $1: 500$ & 30 \\
\hline NGFR & Lab Vision Corp & Chicago, IL & Protease 1 & $1: 50$ & 32 \\
\hline CD57 & BD Biosciences & San Diego, CA & HIER pH 9 & $1: 10$ & 32 \\
\hline CD99 & DAKO & Carpinteria, CA & HIER pH 6 & $1: 5$ & 32 \\
\hline Chromo & In-house (LK2H10) & Ann Arbor, MI & HIER pH 6 & $1: 80$ & 32 \\
\hline Synapto & Biogenex & San Ramon, CA & HIER pH 6 & $1: 600$ & 32 \\
\hline EMA & DAKO & Carpinteria, CA & HIER pH 6 & $1: 25$ & 30 \\
\hline AE1/AE3 & Chemicon Intl & Temecula, CA & Proteinase $\mathrm{K}$ & $1: 100$ & 30 \\
\hline CK7 & DAKO & Carpinteria, CA & Proteinase K & $1: 12.5$ & 30 \\
\hline CK19 & Novocastra & Burlingame, CA & Trypsin 5 min & $1: 150$ & 60 \\
\hline CD56 & Accurate & Westbury, NY & HIER pH 6 & $1: 10$ & 32 \\
\hline bcl-2 & Calbiochem & San Diego, CA & HIER pH 9 & $1: 40$ & 32 \\
\hline Calponin & Novocastra & Burlingame, CA & Proteinase $\mathrm{K}$ & $1: 200$ & 30 \\
\hline Vimentin & DAKO & Carpinteria, CA & HIER pH 6 & $1: 400$ & 30 \\
\hline Beta-catenin & BD Biosciences & San Diego, CA & HIER pH 6 & $1: 500$ & 60 \\
\hline ALK-1 & DAKO & Carpinteria, CA & HIER pH 6 & $1: 50$ & 60 \\
\hline MMP-2 & Novocastra & Burlingame, CA & HIER 1 mM EDTA & $1: 80$ & 60 \\
\hline
\end{tabular}

Chromo, chromogranin; synapto, synaptophysin; SCBT, Santa Cruz Biotechnology; HEIR, heat-induced epitope retrieval (microwave). 
membranous staining, or as membranous when membranous accentuation was present. For betacatenin, positive staining was regarded as any degree of cytoplasmic and/or nuclear staining, however, tumors with nuclear staining were evaluated separately.

Staining patterns were tabulated, and percentages of positive tumors were calculated. Sensitivity, specificity, and positive and negative predictive values were calculated based on any degree of positive staining (weak or strong) in the tumor populations of interest in relation to the study set as a whole. Euclidian (unsupervised) hierarchical cluster analysis of intensity scores was performed using GoldLinker Gold v4.6.1 (Improved Outcome Software, Inveray, ON, Canada) software. Major division points of the data set were placed at the third generation from the most (all) inclusive cluster. Based upon visual assessment of the cluster analysis data and calculated specificity, sensitivity, positive, and negative predictive values, combinations of antibodies deemed most useful in the differential diagnosis for each tumor type were identified.

\section{Results}

\section{Staining Patterns}

Percentages of tumors showing weak or strong staining intensity are presented in Table 2.

\section{Synovial sarcoma}

EMA was the most sensitive marker for synovial sarcoma $(91 \%)$, with $74 \%$ of tumors displaying strong staining. It was also quite specific $(82 \%)$. The cytokeratins as a combined group were $70 \%$ sensitive and $68 \%$ specific. AE1/AE3 was the most sensitive cytokeratin $(70 \%)$, however, displayed staining overlap with the other tumor types, with strong focal staining in $22 \%$ of Ewing sarcomas and $9 \%$ of malignant peripheral nerve sheath tumors. Cytokeratins 7 and 19, although not as sensitive as AE1/AE3, were more specific (100 and 96\%, respectively). Positive staining for both CK7 and EMA in a given tumor was $100 \%$ specific $(52 \%$ sensitive) for synovial sarcoma. Strong staining for bcl-2 or CD56 was present in 48 and $26 \%$ of synovial sarcomas, respectively. Sensitivity/specificity values were $87 / 70 \%$ for bcl-2 and $70 / 88 \%$ for CD56. Based on the cluster map (Figure 1), positivity for these markers tended to be more prevalent in cytokeratin-negative synovial sarcomas. Strong CD99 staining was present in $70 \%$ of synovial sarcomas, including $26 \%$ with strong membranous staining (Figures 2-4).

\section{Malignant peripheral nerve sheath tumor}

S100 had low specificity (54\%) and only moderate sensitivity (57\%) for malignant peripheral nerve sheath tumor. For example, although strong S100

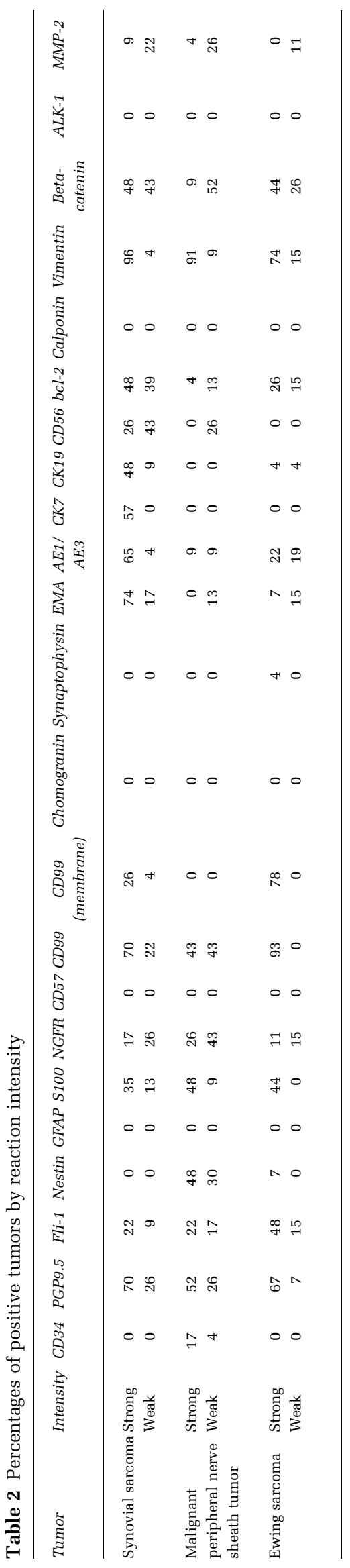


staining was present in $48 \%$ of malignant peripheral nerve sheath tumors, it was also common, although usually focal, in synovial sarcoma (35\%), and Ewing sarcoma $(44 \%)$. NGFR stained more malignant

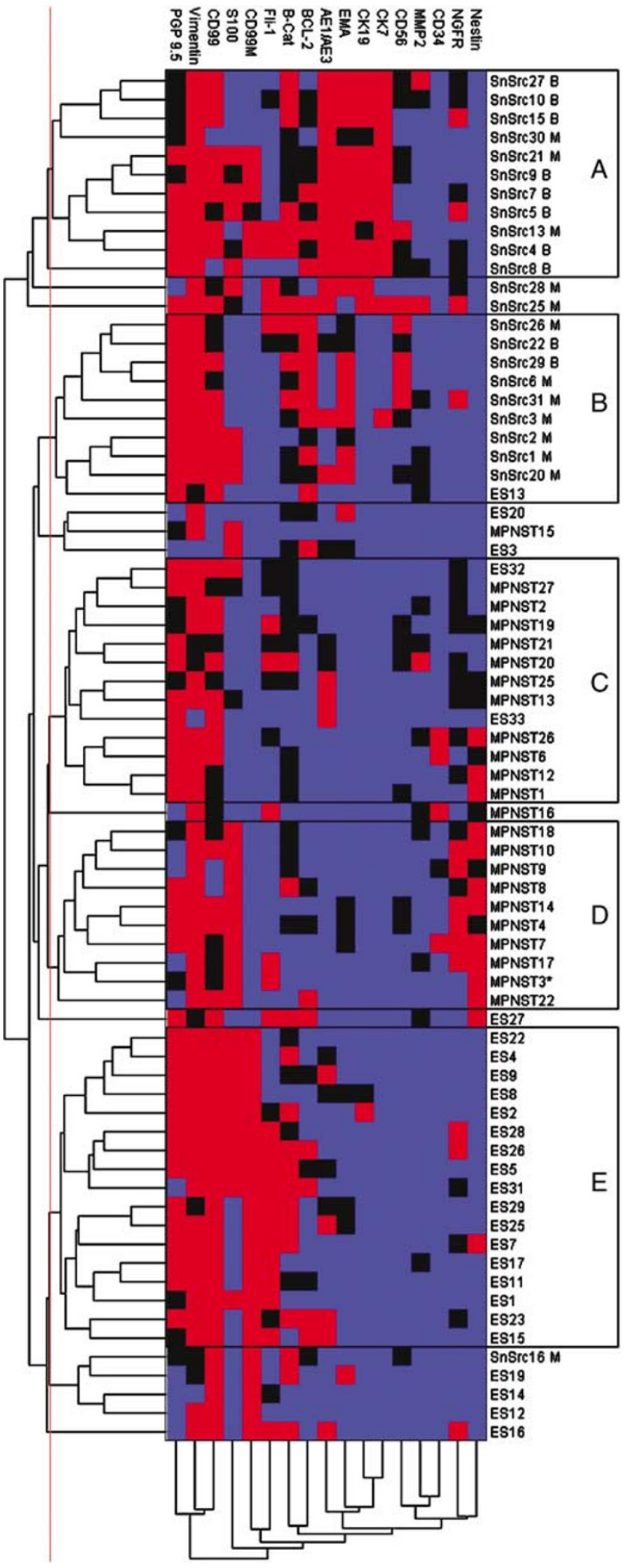

peripheral nerve sheath tumors than did S100, but lacked sufficient sensitivity and specificity to distinguish it from the other tumors. Furthermore, NGFR was often weakly positive, with only $26 \%$ of malignant peripheral nerve sheath tumors showing strong staining. Nestin was the most sensitive $(78 \%)$

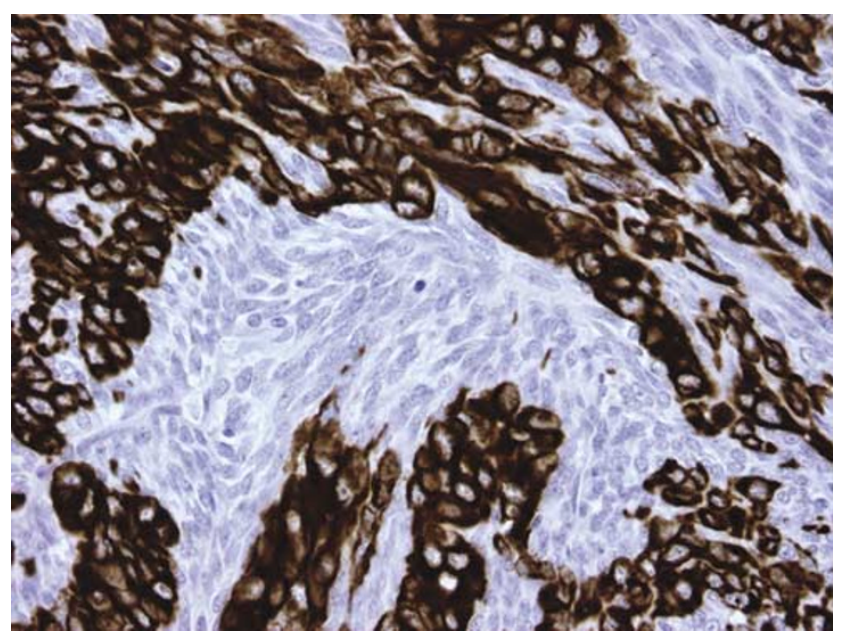

Figure 2 Cytokeratin 7 staining in a biphasic synovial sarcoma. Cytokeratin 7 was highly specific for synovial sarcoma, not present in the other tumors types. The combination of CK7 and EMA had a high degree of specificity and sensitivity for synovial sarcoma, 100 and $52 \%$, respectively.

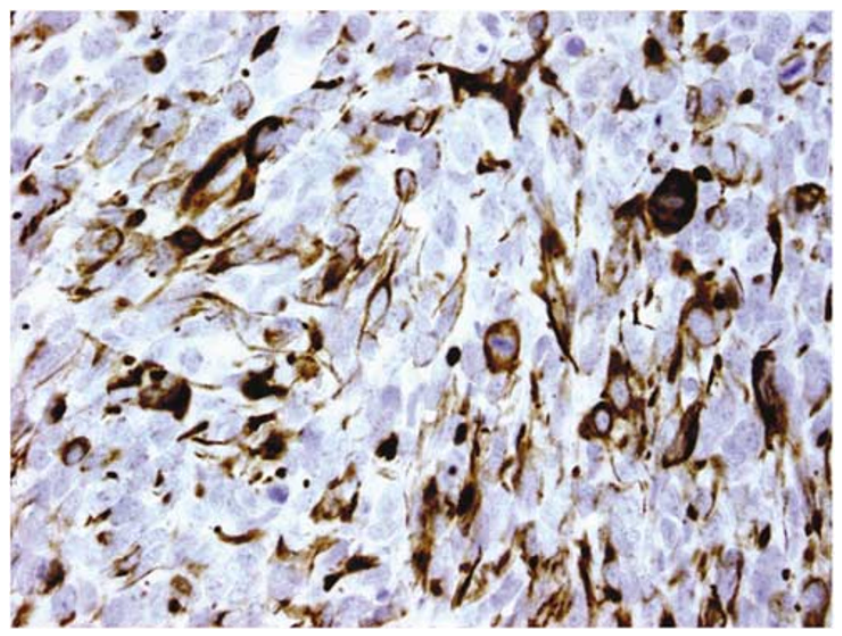

Figure 3 Nestin was the most specific and sensitive marker for malignant peripheral nerve sheath tumor, rarely staining the other tumor types. Combined with S100 the specificity and sensitivity values were 100 and $48 \%$, respectively.

Figure 1 Cluster map of immunohistochemical data. Antibodies are arrayed at the top and tumor types along the right side. Major division points of the data set were placed at the third generation from the most (all) inclusive cluster (vertical red line on left side), which divided the tumors into five clusters, groups A-E. $\mathrm{Red}=$ strongly positive, black $=$ weakly positive, blue $=$ negative, SnSrc $=$ synovial $\quad$ sarcoma,$\quad B=$ biphasic,$\quad \mathrm{M}=$ monophasic, MPNST = malignant peripheral nerve sheath tumor, ES = Ewing sarcoma. 

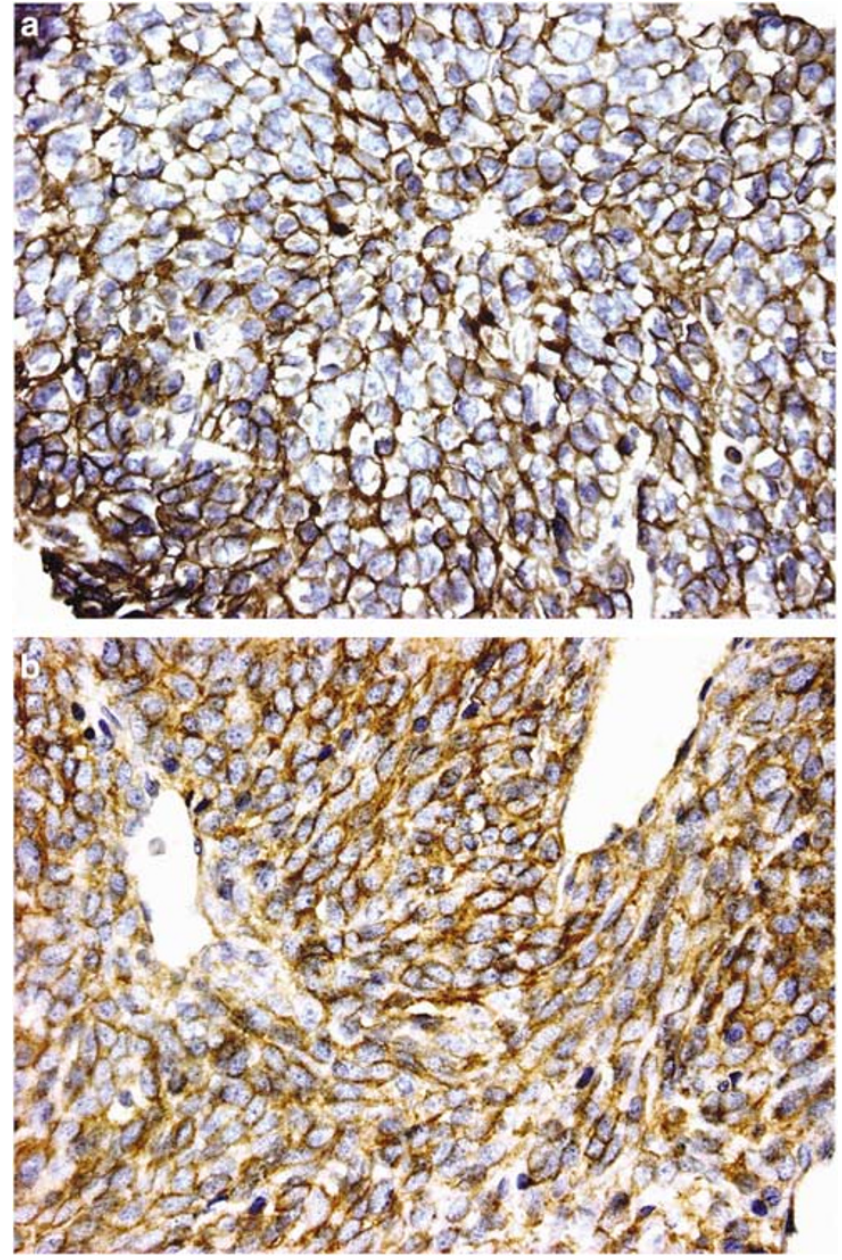

Figure 4 (a) Strong membranous staining for CD99 was present in $78 \%$ of Ewing sarcomas, and was the driving factor in the cluster analysis for this tumor. (b) Membranous CD99 staining, however, showed limited specificity for Ewing sarcoma, exemplified by strong staining in $26 \%$ of synovial sarcomas.

and specific (96\%) stain for malignant peripheral nerve sheath tumor. In addition, its staining intensity tended to be strong, present in $48 \%$ of the tumors. Positivity for both nestin and S100 in a given tumor was highly specific $(100 \%$ specific, $48 \%$ sensitive) for malignant peripheral nerve sheath tumor.

\section{Ewing sarcoma/primitive neuroectodermal tumor}

General (cytosolic or membranous) CD99 staining lacked specificity, and was common in all three tumors. For example, strong staining was present in 93\% of Ewing sarcomas, $70 \%$ of synovial sarcomas, and $43 \%$ of malignant peripheral nerve sheath tumors. Membrane staining was more specific (85\% specificity, 78\% sensitivity) for Ewing sarcoma. However, $26 \%$ of synovial sarcomas also had strong membranous staining, yet all malignant peripheral nerve sheath tumors were negative. Fli-1 stained a majority of Ewing sarcomas (63\% sensitivity, including 48\% with strong nuclear staining). However, it had more limited specificity (65\%), such that $22 \%$ each of synovial sarcomas and malignant peripheral nerve sheath tumors were strongly positive. Positivity for both membranous CD99 and Fli-1 in a given tumor increased the specificity to $96 \%$, with $56 \%$ sensitivity.

\section{Nonspecific and nonsensitive antibodies}

Vimentin and PGP9.5 showed low specificities, each staining many tumors of each type. Similarly, MMP2 and beta-catenin stained various tumors, but did not display distinct patterns. Strong nuclear staining for beta-catenin was present in $13 \%$ of synovial sarcomas, $4 \%$ of malignant peripheral nerve sheath tumors, and $0 \%$ of Ewing sarcomas. Chromogranin, CD57, ALK-1, GFAP, CD34, and calponin displayed no staining in any tumor, while synaptophysin was positive in only one Ewing sarcoma in this study.

\section{Cluster Analysis}

Cluster analysis highlighted five main groups (Figure 1) of related staining patterns, and in general, like tumor types clustered together. However, overlap of staining patterns caused three Ewing sarcomas to be included within synovial sarcoma and malignant peripheral nerve sheath tumor groups, and 13 other tumors of various types to be outliers, not falling into any defined group.

\section{Synovial sarcoma}

Synovial sarcomas generally fell into two groups, A and B. Group A $(n=11)$ was based on uniform positivity for EMA and one or more of the cytokeratin subtypes, and consisted entirely of synovial sarcomas. Eight (72\%) were biphasic and three $(27 \%)$ were monophasic. Ten $(91 \%)$ group A tumors had an $S S X 1$ translocation, while only one $(9 \%)$ had $S S X 2$. Five (45\%) of these group A tumors demonstrated strong membranous CD99 staining. Strong bcl-2 staining was present in three $(27 \%)$ tumors, while strong CD56 staining was seen in only one. Group B synovial sarcomas $(n=9)$ were also uniformly positive for EMA, but rarely positive for cytokeratin, with only two cases $(22 \%)$ showing strong staining for AE1/AE3. Among the nine tumors in this group, two $(22 \%)$ were biphasic and seven $(78 \%)$ were monophasic. Six $(67 \%)$ were SSX1 and three (33\%) were SSX2. Compared to Group A, the tumors in group B demonstrated higher rates of strong staining for bcl-2 $(78 \%)$ and CD56 (44\%). Although all of these tumors were CD99 positive, none had membranous accentuation. Group B also included a CD99-positive Ewing sarcoma that lacked membranous accentuation (ES 13 in Figure 1). This tumor, confirmed by FISH, was strongly positive for bcl-2, but lacked any EMA or CK staining. Three synovial sarcomas were outliers that failed to cluster with any group, two of which 
(SnSrc 25 and 28 in Figure 1) were fairly closely related to groups A and B. Both were monophasic, SSX2 synovial sarcomas that were positive for cytokeratin and CD99, but negative for membranous CD99; one was negative for EMA. The third synovial sarcoma outlier (SnSrc 16) was more distant in the cluster histogram and actually was more closely related to Ewing sarcoma (group E). This monophasic, SSX1 tumor was strongly positive for membranous CD99, negative for EMA and cytokeratin, and showed only weak staining for bcl-2 and CD56.

\section{Malignant peripheral nerve sheath tumor}

Most of the malignant peripheral nerve sheath tumors similarly fell into two groups, C and D. Group C $(n=13)$ contained tumors that were predominantly negative for S100 with inconsistent and mostly weak staining for nestin and NGFR. This group included two Ewing sarcomas (ES 32 and 33 in Figure 1), both with cytosolic, but not membranous, CD99 staining; one confirmed by FISH, the other non-informative. Group D $(n=10)$ consisted entirely of malignant peripheral nerve sheath tumors that were uniformly S100 positive. Strong staining for nestin and NGFR were present in eight $(80 \%)$ and five $(50 \%)$ tumors, respectively. As the majority (96\%) of malignant peripheral nerve sheath tumors in our study set were spindle cell type (with only one epithelioid malignant peripheral nerve sheath tumor), no correlation could be made between histology and immunohistochemical profile. Two malignant peripheral nerve sheath tumor outliers failed to cluster into a defined group. One of these (MPNST 16) was close in relation to group C and was similarly S100 negative, only weakly positive for nestin, and negative for NGFR. The second tumor (MPNST 15) was S100 positive, but negative for both nestin and NGFR. Seven (64\%) group C tumors, three (30\%) group D tumors, and one outlier occurred in neurofibromatosis patients.

\section{Ewing sarcoma/primitive neuroectodermal tumor} Most Ewing sarcomas fell into a single cluster, group E $(n=17)$ based upon strong membranous CD99 staining in all the tumors $(100 \%)$ and strong staining for Fli-1 in 11 (65\%). This group consisted entirely of Ewing sarcomas. Thirteen of these tumors were confirmed by FISH, one was negative, and three were considered noninformative. As noted above, one Ewing sarcoma (ES 13), which was CD99 positive without membranous accentuation and Fli-1 negative, clustered with synovial sarcomas into group B. Two Ewing sarcomas (ES 32 and 33), which were also CD99 positive without membranous accentuation, clustered with malignant peripheral nerve sheath tumors into group C (ES 32 confirmed by FISH, ES 33 noninformative). Seven (26\% of the Ewing sarcomas) were outliers that failed to cluster with any group. Two of these (ES 3 and 20) were CD99- and Fli-1 negative. One (ES 27) was CD99 positive but Fli-1 negative. Four (ES 12, 14,16 , and 19) were membranous CD99 positive and Fli-1 negative. All seven Ewing sarcoma outliers were confirmed by FISH.

\section{Discussion}

Cluster analysis is a statistical methodology, which organizes complex multivariate data sets into groups or clusters with similar patterns. Multiple types of cluster analysis exist and have been used in a wide variety of fields, but a particularly relevant example is the hierarchical cluster analysis employed in analysis of gene or DNA microarray data ${ }^{30}$ In short, hierarchichical analysis works by grouping similar objects into small clusters and then successively merging clusters until one large cluster comprising the whole data set is achieved. The clustering is then displayed in the form of a branching tree diagram, which can be broken into distinct clusters by cutting across the tree at a particular height. In this study, we used GoldLinker Gold v4.6.1 (Improved Outcome Software, Inveray, ON, Canada) software to perform an unsupervised (raw data without preset classifications) hierarchical cluster analysis of our manually scored immunohistochemical data. We analyzed a large data set of 22 antibodies in 73 tumors consisting of three sarcoma phenotypes that have overlapping morphologies. Using a model (tissue microarray cores) that mimics small biopsy sampling, cluster analysis was very effective at grouping the tumors into diagnostic categories, for graphically identifying specific antibodies that drive the differential clustering that can be confirmed by statistical calculations, and for designing useful antibody panels. In addition, it highlighted a number of important caveats inherent to the immunohistochemical differential diagnosis of these tumors.

Synovial sarcoma is relatively easy to diagnose when it shows classic biphasic morphology and/or when it is cytokeratin- or EMA positive. Diagnostic difficulties arise, however, with monophasic and poorly differentiated tumors, as well as in small samplings of biphasic tumors comprising only the spindle cell component. In addition, it is not uncommon for synovial sarcoma to be cytokeratinor EMA negative in core biopsies. Since the morphologic features in synovial sarcoma are quite variable, it can mimic other tumors, especially small round cell tumors ${ }^{9}$ such as Ewing sarcoma, rhabdomyosarcoma, and mesenchymal chondrosarcoma; or pure spindle cell tumors such as malignant peripheral nerve sheath tumor and fibrosarcoma. Immunohistochemically, synovial sarcoma can also mimic Ewing sarcoma and malignant peripheral nerve sheath tumor since it can be positive for CD99 or S100,,$^{1,8,9}$ respectively.

In this study, EMA was the single most sensitive and specific marker for synovial sarcoma, staining 
all but two cases. We also found cytokeratin stains in general to be specific, although not quite as sensitive as EMA. As previously reported, certain cytokeratin subsets, ${ }^{3,9,14}$ notably CK7 and CK19, show greater specificity than pan-keratins such as AE1/AE3. We found CK7 to be the most specific marker for synovial sarcoma, demonstrating strong staining in over half the cases, yet with no staining in any of the other tumor types. CK19 was only slightly less specific. In contrast, AE1/AE3 stained multiple Ewing sarcomas and malignant peripheral nerve sheath tumors, a finding observed by others, ${ }^{4,6,14}$ and is therefore less helpful diagnostically. The combination of positive staining for both EMA and CK7 staining in a given tumor is highly specific ( $100 \%$ in this study) with a high positive predictive value for distinguishing synovial sarcoma from malignant peripheral nerve sheath tumor and Ewing sarcoma.

Although a majority of the synovial sarcomas stained with bcl-2 and to a lesser extent CD56, strong staining was present in only 48 and $26 \%$, respectively. However, these markers showed rather high specificities, and therefore, bcl-2 and CD56 appear to be well suited as adjuncts or second tier stains to EMA and cytokeratin. In this study, bcl-2 and CD56 were especially useful in cytokeratinnegative tumors. Many of the synovial sarcomas in this study were CD99 positive, with a substantial number $(26 \%)$ showing strong membranous staining, often regarded as a more or less specific pattern for Ewing sarcoma. ${ }^{10}$ Although, CD99 staining in synovial sarcoma is well known ${ }^{1,9}$ and even serves as an adjunctive diagnostic marker, this highlights an important potential pitfall with CD99. It is important to be aware of CD99 staining in synovial sarcoma since it can resemble a small round cell tumor and be misdiagnosed as Ewing sarcoma with a limited antibody panel. On the other hand, membranous CD99 staining in synovial sarcoma may actually be helpful in differentiatiating monophasic or poorly differentiated synovial sarcoma from morphologically similar malignant peripheral nerve sheath tumor, since no malignant peripheral nerve sheath tumor stained with a membranous pattern in our study set. Other purportedly useful markers of synovial sarcoma, beta-catenin, MMP-2, and calponin, ${ }^{16-18}$ were seemingly useless in our data set. For example, beta-catenin marked significant numbers of both malignant peripheral nerve sheath tumors and Ewing sarcomas and thus had low specificity. When analysis was restricted to nuclear beta-catenin staining, strong reactivity was present in only three synovial sarcomas and one malignant peripheral nerve sheath tumor, which had no effect on the cluster analysis. MMP-2 stained only scattered tumors throughout the data set, and calponin stained no tumors.

Cluster analysis divided synovial sarcomas into two groups with different morphologic and molecular tendancies. The first group, driven by positivity for both EMA and cytokeratin, was dominated by biphasic (73\%) and SSX1 translocated tumors (91\%). The second group, which consisted of EMA-positive and mostly cytokeratin-negative tumors, was dominated by monophasic synovial sarcomas $(78 \%)$, and more SSX2 translocated tumors $(33 \%)$.

In contrast to synovial sarcoma, malignant peripheral nerve sheath tumor is notoriously difficult to diagnose, forming several different morphologic patterns, including spindle cell tumors that resemble synovial sarcoma, a small cell variant mimicking Ewing sarcoma, ${ }^{31}$ as well as epithelioid and pleomorphic patterns. S100 is traditionally regarded as the best marker for malignant peripheral nerve sheath tumor, however, it is positive in only around half the tumors. ${ }^{7,22}$ We found $48 \%$ of malignant peripheral nerve sheath tumors to be strongly positive in our study. Furthermore, S100 also stains synovial sarcoma ${ }^{8}$ and Ewing sarcoma, ${ }^{32}$ exemplified by 35 and $44 \%$, respectively, with strong staining in our study set. Other reportedly helpful markers, CD34 ${ }^{22}$ and GFAP, ${ }^{21}$ displayed little utility in our study set. CD34 stained only scattered tumors, while GFAP stained none. CD57, another purportedly useful marker, failed to stain any of our malignant peripheral nerve sheath tumors, which was curious, considering the relatively high percentages of staining that have been reported.7,21,22 The reason for this difference is not clear, since we had excellent internal and external controls (tonsillar germinal center natural killer cells), and we repeated the staining at a lower titer with the same results.

We evaluated a number of other markers of neural differentiation (NGFR, nestin, and PGP9.5), as potentially useful in malignant peripheral nerve sheath tumor. Although NGFR stained the majority of malignant peripheral nerve sheath tumors in our study set, it mostly displayed weak staining, with strong staining in only $26 \%$. In addition, NGFR had low specificity, particularly due to staining in nearly half the synovial sarcomas, a finding previously reported. ${ }^{9,33,34}$ PGP9.5 was not useful since it stained high numbers of all three tumors types in this study. The lack of specificity for this marker has been highlighted in the literature. ${ }^{35}$

Nestin proved to be the single best marker for malignant peripheral nerve sheath tumor in this study, showing high specificity and sensitivity values (96 and $78 \%$, respectively). Strong staining for nestin was present in $48 \%$ of tumors, compared to strong staining in only one Ewing sarcoma and no synovial sarcomas. Positive staining for both S100 and nestin in a given tumor was highly specific with a positive predictive value of $100 \%$ for distinguishing malignant peripheral nerve sheath tumor from synovial sarcoma and Ewing sarcoma. As with synovial sarcoma, cluster analysis divided malignant peripheral nerve sheath tumor into two groups. One group was immunohistochemically well differentiated consisting entirely of S100- and 
nestin-positive tumors, while the other group was largely negative for these markers. We were unable to identify any morphological differences between these two groups. However, since the vast majority of malignant peripheral nerve sheath tumors in our study set were spindle cell tumors, we are unable to comment about differential staining patterns in the various morphologic subtypes of malignant peripheral nerve sheath tumor. And, while we found a stronger association between neurofibromatosis and the less differentiated group $\mathrm{C}$ tumors, the significance of this result is unclear. Importantly, there still exists a relatively large group of poorly differentiated S100-negative malignant peripheral nerve sheath tumors, which continues to be difficult to diagnose by immunohistochemistry. With the addition of nestin to the panel, this group can be reduced.

Ewing sarcoma historically had been defined by absence of a specific line of differentiation, and immunohistochemical studies were primarily carried out to exclude other small round cell tumors, especially lymphoma and rhabdomyosarcoma. When CD99 was discovered it was initially believed to be a specific Ewing marker. ${ }^{36}$ However, it was found to stain other tumors, including lymphoblastic lymphoma, ${ }^{37}$ mesenchymal chondrosarcoma, ${ }^{13}$ and synovial sarcoma ${ }^{1,9}$ among others, which are within the morphologic differential diagnosis of Ewing sarcoma. A membranous pattern of CD99 staining is believed to be more specific than cytosolic staining, ${ }^{10}$ and although it does appear to be more specific, this too can be found in other tumors. For instance, $26 \%$ of synovial sarcomas in our study had strong membranous CD99 staining. By contrast, although $43 \%$ of malignant peripheral nerve sheath tumors had strong CD99 staining, none had a membranous pattern, suggesting that the pattern of CD99 staining may have some utility for differentiating Ewing sarcoma from it.

Fli-1, the gene product of $\mathrm{t}(11: 22)$, initially reported to be useful in Ewing sarcoma, ${ }^{38,39}$ was similarly found to stain other tumors, including lymphoblastic lymphoma. ${ }^{39}$ In our study, it had moderate sensitivity $(68 \%)$, similar to reported values, ${ }^{38,39}$ but limited specificity, displaying overlap staining with both synovial sarcoma and malignant peripheral nerve sheath tumor, $22 \%$ each with strong nuclear staining. Thus, Fli-1 is probably most useful in combination with a panel of markers, serving as an adjunct to CD99. The neuroectodermal marker PGP9.5 lacks specificity, staining multiple synovial sarcomas and malignant peripheral nerve sheath tumors, ${ }^{35}$ and is therefore not useful. Chromogranin and synaptophysin showed virtually no staining in our Ewing sarcomas; most likely due to a disproportionate number of undifferentiatied (nonprimitive neuroectodermal) tumors in the data set.

Based upon visual examination of the cluster analysis data along with calculated sensitivity, specificity, positive and negative predictive values, we were able to identify pairs of antibodies for each tumor type (EMA/CK7 for synovial sarcoma, S100/ nestin for malignant peripheral nerve sheath tumor, and CD99/Fli-1 for Ewing sarcoma) that were highly specific within this differential diagnostic context. Based upon our results we would recommend this panel of six markers. CD56 and bcl-2 can be added when synovial sarcoma is suspected but EMA and CK7 are negative.

By grouping tumors on the basis of their immunohistochemical profiles, hierarchical cluster analysis displays the data points in a visually manageable format from which one can make observations, draw conclusions, and design antibody panels. Additionally, the small tissue samplings in the tissue microarrays as a model mimic core biopsy specimens. In this study, we preselected three sarcomas that have overlapping morphologic and immunohistochemical features and stained them with a large panel of markers regarded as useful in the differential diagnosis. Cluster analysis grouped the tumors into five groups with very little overlap of tumor types. It highlighted combinations of antibodies that appear most useful in the differential diagnoses, which were confirmed by statistical calculations. It also reaffirmed the shortcomings and pitfalls of immunohistochemistry, exemplified by a number of outliers (ie tumors which did not cluster into any of the defined groups), as well as a few tumors that showed aberrant clustering. Hierarchical cluster analysis is a very effective method for analyzing and comparing large sets of immunohistochemical data.

\section{Acknowledgements}

This work was supported in part by the Robert Urich Memorial Sarcoma Fund at the University of Michigan. Dr Thomas is a Walther Cancer Institute Research Investigator and acknowledges their continued support. We thank Dr Christine E Fuller, St Jude Children's Research Hospital, Memphis, $\mathrm{TN}$, for performing fluorescence in situ hybridization in the Ewing sarcoma cases.

\section{References}

1 Dei Tos AP, Wadden C, Calonje E. Immunohistochemical demonstration of glycoprotein p30/32MIC2 (CD99) in synovial sarcoma: a potential cause of diagnostic confusion. Appl Immunohistochem 1995;3:168-173.

2 Ordonez NG, Mahfouz SM, Mackay B. Synovial sarcoma: an immunohistochemical and ultrastructural study. Hum Pathol 1990;21:733-749.

3 Miettinen M, Limon J, Niezabitowski A, et al. Patterns of keratin polypeptides in 110 biphasic, monophasic, and poorly differentiated synovial sarcomas. Virchows Arch 2000;437:275-283. 
4 Smith TA, Machen SK, Fisher C, et al. Usefulness of cytokeratin subsets for distinguishing monophasic synovial sarcoma from malignant peripheral nerve sheath tumor. Am J Clin Pathol 1999;112:641-648.

$5 \mathrm{Gu}$ M, Antonescu CR, Guiter G, et al. Cytokeratin immunoreactivity in Ewing's sarcoma: prevalence in 50 cases confirmed by molecular diagnostic studies. Am J Surg Pathol 2000;24:410-416.

6 Folpe AL, Goldblum JR, Rubin BP, et al. Morphologic and immunophenotypic diversity in Ewing family tumors: a study of 66 genetically confirmed cases. Am J Surg Pathol 2005;29:1025-1033.

7 Meis JM, Enzinger FM, Martz KL, et al. Malignant peripheral nerve sheath tumors (malignant schwannomas) in children. Am J Surg Pathol 1992;16:694-707.

8 Fisher C, Schofield JB. S-100 protein positive synovial sarcoma. Histopathology 1991;19:375-377.

9 Folpe AL, Schmidt RA, Chapman D, et al. Poorly differentiated synovial sarcoma: immunohistochemical distinction from primitive neuroectodermal tumors and high-grade malignant peripheral nerve sheath tumors. Am J Surg Pathol 1998;22:673-682.

10 Sebire NJ, Gibson S, Rampling D, et al. Immunohistochemical findings in embryonal small round cell tumors with molecular diagnostic confirmation. Appl Immunohistochem Mol Morphol 2005;13:1-5.

11 Lucas DR, Bentley G, Dan ME, et al. Ewing sarcoma vs lymphoblastic lymphoma. A comparative immunohistochemical study. Am J Clin Pathol 2001;115:11-17.

12 Devaney K, Abbondanzo SL, Shekitka KM, et al. MIC2 detection in tumors of bone and adjacent soft tissues. Clin Orthop 1995;310:176-187.

13 Granter SR, Renshaw AA, Fletcher CD, et al. CD99 reactivity in mesenchymal chondrosarcoma. Hum Pathol 1996;27:1273-1276.

14 Machen SK, Fisher C, Gautam RS, et al. Utility of cytokeratin subsets for distinguishing poorly differentiated synovial sarcoma from peripheral primitive neuroectodermal tumour. Histopathology 1998;33: 501-507.

15 Hirakawa N, Naka T, Yamamoto I, et al. Overexpression of bcl-2 protein in synovial sarcoma: a comparative study of other soft tissue spindle cell sarcomas and an additional analysis by fluorescence in situ hybridization. Hum Pathol 1996;27:1060-1065.

16 Fisher C, Montgomery E, Healy V. Calponin and h-caldesmon expression in synovial sarcoma; the use of calponin in diagnosis. Histopathology 2003;42: 588-593.

17 Hasegawa T, Yokoyama R, Matsuno Y, et al. Prognostic significance of histologic grade and nuclear expression of beta-catenin in synovial sarcoma. Hum Pathol 2001;32:257-263.

18 Saito T, Oda Y, Sakamoto A, et al. Matrix metalloproteinase-2 expression correlates with morphological and immunohistochemical epithelial characteristics in synovial sarcoma. Histopathology 2002;40: 279-285.

19 Hoshi N, Hiraki H, Yamaki T, et al. Frequent expression of $75 \mathrm{kDa}$ nerve growth factor receptor and phosphotyrosine in human peripheral nerve tumours: an immunohistochemical study on paraffin-embedded tissues. Virchows Arch 1994;424:563-568.

20 Shimada STT, Nagasaka T, Kuroda M, et al. Nestin expression in malignant peripheral nerve sheath tumor (MPNST) and other soft tissue tumors. Mod Pathol 2005;1:22A.
21 Giangaspero F, Fratamico FC, Ceccarelli C, et al. Malignant peripheral nerve sheath tumors and spindle cell sarcomas: an immunohistochemical analysis of multiple markers. Appl Pathol 1989;7:134-144.

22 Zhou H, Coffin CM, Perkins SL, et al. Malignant peripheral nerve sheath tumor: a comparison of grade, immunophenotype, and cell cycle/growth activation marker expression in sporadic and neurofibromatosis 1-related lesions. Am J Surg Pathol 2003;27:13371345.

23 Hoang MP, Sinkre P, Albores-Saavedra J. Expression of protein gene product 9.5 in epithelioid and conventional malignant peripheral nerve sheath tumors. Arch Pathol Lab Med 2001;125:1321-1325.

24 Parham DM, Hijazi Y, Steinberg SM, et al. Neuroectodermal differentiation in Ewing's sarcoma family of tumors does not predict tumor behavior. Hum Pathol 1999;30:911-918.

25 Dierick AM, Roels H, Langlois M. The immunophenotype of Ewing's sarcoma. An immunohistochemical analysis. Pathol Res Pract 1993;189:26-32.

26 Banerjee SS, Agbamu DA, Eyden BP, et al. Clinicopathological characteristics of peripheral primitive neuroectodermal tumour of skin and subcutaneous tissue. Histopathology 1997;31:355-366.

27 Carter RL, al-Sams SZ, Corbett RP, et al. A comparative study of immunohistochemical staining for neuronspecific enolase, protein gene product 9.5 and S-100 protein in neuroblastoma, Ewing's sarcoma and other round cell tumours in children. Histopathology 1990;16:461-467.

28 Thomas DG, Giordano TJ, Sanders D, et al. Expression of receptor tyrosine kinases epidermal growth factor receptor and HER-2/neu in synovial sarcoma. Cancer 2005;103:830-838.

29 Lai R, Rodriguez-Galindo C, Navid F, et al. STAT3 activation in ewing sarcoma family of tumors. Mod Pathol 2005;18:17A.

30 Yeung KY, Fraley C, Murua A, et al. Model-based clustering and data transformations for gene expression data. Bioinformatics 2001;17:977-987.

31 Abe S, Imamura T, Park P, et al. Small round-cell type of malignant peripheral nerve sheath tumor. Mod Pathol 1998;11:747-753.

32 Franchi A, Pasquinelli G, Cenacchi G, et al. Immunohistochemical and ultrastructural investigation of neural differentiation in Ewing sarcoma/PNET of bone and soft tissues. Ultrastruct Pathol 2001;25: 219-225.

33 Perosio PM, Brooks JJ. Expression of nerve growth factor receptor in paraffin-embedded soft tissue tumors. Am J Pathol 1988;132:152-160.

34 Fanburg-Smith JC, Miettinen M. Low-affinity nerve growth factor receptor (p75) in dermatofibrosarcoma protuberans and other nonneural tumors: a study of 1150 tumors and fetal and adult normal tissues. Hum Pathol 2001;32:976-983.

35 Campbell LK, Thomas JR, Lamps LW, et al. Protein gene product 9.5 (PGP 9.5) is not a specific marker of neural and nerve sheath tumors: an immunohistochemical study of 95 mesenchymal neoplasms. Mod Pathol 2003;16:963-969.

36 Ambros IM, Ambros PF, Strehl S, et al. MIC2 is a specific marker for Ewing's sarcoma and peripheral primitive neuroectodermal tumors. Evidence for a common histogenesis of Ewing's sarcoma and peripheral primitive neuroectodermal tumors from MIC2 
expression and specific chromosome aberration. Cancer 1991;67:1886-1893.

37 Riopel M, Dickman PS, Link MP, et al. MIC2 analysis in pediatric lymphomas and leukemias. Hum Pathol 1994;25:396-399.

38 Llombart-Bosch A, Navarro S. Immunohistochemical detection of EWS and FLI-1 proteinss in Ewing sarcoma and primitive neuroectodermal tumors: comparative analysis with CD99 (MIC-2) expression. Appl Immunohistochem Mol Morphol 2001;9:255-260.

39 Folpe AL, Hill CE, Parham DM, et al. Immunohistochemical detection of FLI-1 protein expression: a study of 132 round cell tumors with emphasis on CD99-positive mimics of Ewing's sarcoma/primitive neuroectodermal tumor. Am J Surg Pathol 2000;24: 1657-1662. 